\title{
Effectiveness and Implication of Sensory Integration Therapy on School Performance of Children with Learning Disabilities
}

\author{
Sonia N Young* and Karen Furgal \\ Doctor of Physical Therapy Program, College of Health and Human Services, Western Kentucky University, Bowling Green, Kentucky, USA
}

"Corresponding author: Sonia N Young, Doctor of Physical Therapy Program, College of Health and Human Services, Western Kentucky University, Bowling Green, Kentucky, USA, Tel: (270) 745-3233; Fax: (270) 745-3497; E-mail: sonia.young@wku.edu

Rec date: Jan 31, 2016; Acc date: Feb 23, 2016; Pub date: Feb 29, 2016

Copyright: ( 2016 Young SN, et al. This is an open-access article distributed under the terms of the Creative Commons Attribution License, which permits unrestricted use, distribution, and reproduction in any medium, provided the original author and source are credited.

\section{Introduction}

School aged children with learning disabilities present unique challenges and opportunities for school professionals. The National Joint Commission on Learning Disabilities (LD) contend that these learning disabilities are often present early and continue throughout the lifespan, with a change in severity at different stages [1]. These children often have accompanying sensory integration dysfunction (SID) when they are unable to fully process and integrate sensory information. Katz defines sensory integration as "an innate neurobiological process in which the brain integrates and interprets sensory stimuli in the environment" and further contends that when children are not able to fully process and integrate the sensory information coming to them from their environment, they can become over or under stimulated leading to academic or behavioral difficulties [2]. Additionally, children diagnosed with autism and developmental coordination disorder (DCD) have demonstrated SID [3] which falls within the 2016 ICD-10- CM Diagnostic code of F88 "Other disorders of psychological development" within the Diagnostic Related Group of 886 "Behavioral and developmental disorders" [4]. One treatment of SID is Sensory Integration Therapy (SIT) which was first developed by A. Jean Ayers, an occupational therapist with advanced training in educational psychology and neuroscience, and published in the 1970s [5]. Children with SID can receive SIT in the classroom by physical and occupational therapists [6], however, this can be a challenging treatment model to integrate into the school setting due to specialized equipment required. The therapists work with teachers and counselors to provide an appropriate learning environment for the students by addressing these impairments sensory and motor deficits associated with SID, while collaborating to make modifications and accommodations for better school performance [7]. This editorial provides a brief glimpse into the effectiveness and practical implications of SIT for various school-based populations.

\section{Effectiveness}

Earlier studies both support and refute the effectiveness of sensory integration therapy (SIT) in the treatment of children with $\operatorname{LD}[8,9]$. More recent studies also show mixed results [10,11]. A meta-analysis of research on SIT for individuals with developmental and learning disorders reported that a small effect occurred when SIT techniques were compared with no treatment, but no significant effect was noted when compared with alternative interventions [10]. Systematic reviews of sensory-based interventions in individuals with autism explored the difference between SIT (performed in a specialized clinic) and sensory-based interventions (individual techniques performed within a daily routine) and again found mixed results, with either small effects from sensory-based activities or no effect at all $[11,12]$.
A pilot study was conducted in Japan that compared SIT and Group Therapy (GT) in high functioning autistic individuals [13]. SIT was provided by an occupational therapist in a therapy room equipped with sensory and kinetic equipment. The GT program included activities from multiple domains (communication, social, motor) in small groups of 5-6 children. An occupational therapist, speech therapist and three nursery school teachers administered this technique. The findings include a statistically significant increase in scores on the Japanese version of the Miller Assessment for Preschoolers in the SIT group [13]. While this study and others like it show promise in helping to define what works and what does not, the efficacy on individuals who have a diagnosis of autism and are lower functioning should be further explored.

Children with Autism Spectrum Disorder (ASD) have noted difficulty integrating and processing sensory information [14] including difficulty integrating speech and interpreting the related social context [15]. A randomized trial of school aged children with ASD compared use of a manualized occupational therapy/sensory integration intervention program developed by Schaaf et al. based on the Ayres SIT techniques, to a usual care control group $(n=15)$ [14]. Intervention to the experimental group $(n=17)$ was provided three times per week for 10 weeks in one hour sessions. Children in the experimental group obtained significantly higher scores on the primary outcome measure (Goal Attainment Scales) $(\mathrm{p}=0.003, \mathrm{~d}=1.2)$ and with 2 subtests of the secondary outcome measure (Pediatric Evaluation of Disability Inventory: self-care $(\mathrm{p}=0.008, \mathrm{~d}=0.9)$ and socialization $(\mathrm{p}=0.04, \mathrm{~d}=0.7)$ [14].

Difficulty in utilizing SIT in school-based therapy has led some therapists to utilize Sensory Based Interventions (SBI) in schools. Differences between SIT and SBI include location, child versus adultdirected modalities, and adaptability to routine. Location of SIT most often occurs in a center-based facility with specialized equipment (specialized swings, trampolines, climbing walls) that is set up by the therapist to be in line with the child's skill set, but child-directed in the sense that the child is able to self-select activities within that framework. In the case of SBI, modalities are typically applied to the child as they fit into the daily routine. Routine-based activities have included brushing, massage, swinging, bouncing or wearing a vest. These activities, although more structured to fit within the schoolbased framework, are in line with the notion that the various sensory systems must be activated to organize the sensory system [11].

Additional strategies exist to treat children with SID other than SIT and SBI. These include behavioral interventions as well as alternate strategies such as sound-based intervention like The Listening Program $^{\circ}$ [16] and developmental programs such as the Developmental, Individual Difference, Relationship-based (DIR $\left.{ }^{\circ}\right) /$ Floortime $^{\mathrm{TM}}$ model [17]. Children with SPD can have difficulty 
controlling or have a quicker and more intense response to a typical sensory stimulus [16]. As a result, negative reactions to auditory or tactile input can be present and lead to behavioral problems in school. The Listening Program ${ }^{\star}$ can be implemented by occupational therapists and utilizes modified music delivered through use of headphones and an amplifier to address the over-stimulation of the sensory system. This program has been effective in decreasing negative and self-stimulating behaviors [16]. The DIR $/$ Floortime $^{\mathrm{TM}}$ model was first published in 2000 and incorporates use of relationships and play to improve a child's emotional developmental and sensory processing [17].

While research results are varied due in part to methodological limitations, physical and occupational therapists and school professionals continue to utilize and see results in the classroom from selected SI theory-based techniques. The effectiveness of SI in isolation can be challenging to demonstrate through research findings because children with SID often have multiple physical, behavioral, and learning disabilities that require a variety of individualized interventions that are applied simultaneously to treat the complex problems that are occurring [8]. This makes it difficult to design studies that account for these multiple variables and utilize standardized protocols.

\section{Practical Implications}

For children who have SID with related learning disabilities, SI techniques can be applied in the classroom or school to improve sensory processing and integration. Children who have difficulty processing sensory information have demonstrated difficulty focusing in the classroom [7]. Lack of focus and frustration from an inability to integrate environmental stimuli can lead to disruptive behaviors in the classroom. Learning may be difficult if these students are not identified and managed appropriately. Teachers and counselors should be trained to identify symptoms of SI disorders because the manifestations of them are often misinterpreted as emotional or behavioral disorders [2]. Timely referral to a physical or occupational therapist for further assessment and intervention will increase the likelihood that appropriate management strategies, including of application of SIT in the classroom, will be provided. These professionals can work collaboratively in the classroom to enable a more effective learning environment for the student.

Management strategies include application of sensory-based techniques and environmental modifications. Common sensory-based techniques that have been applied and studied in a school-based setting include: use of a scheduled sensory activities that may include use of a therapy ball in lieu of a regular classroom chair, wearing a weighted or compression vest, and intermittent use of brushing, swinging, and jumping. These activities are typically recommended and monitored by the school occupational therapist, and may be supported by the physical therapist [10]. Modifications in the classroom include decreasing sensory distractions by limiting extraneous sights and sounds, thereby reducing the likelihood of overstimulation [18]. SIT in a school based setting can be a feasible strategy if school administrators are supportive and willing to allocate space and resources for specialized equipment. Additionally, an SItrained occupational therapist is needed to appropriately direct the utilization of these resources.
A physical therapist is a supportive member of a collaborative team and can be a valuable resource to help meet the needs of children with SID. Educators can improve services to children with LD and SID by providing appropriate early identification, classroom modifications, counseling, and treatment plans. SIT can be an effective clinical tool to use with children with SID and LD; however, continued research is warranted to confirm these findings.

\section{References}

1. (2016) National Joint Commission: Operationalizing the NJCLD Definition of Learning Disabilities for Ongoing Assessment in School.

2. Katz I (2006) Students with sensory integration dysfunctions: Issues for school counselors. Journal of School Counseling 4: 1-22.

3. Barnhart RC, Davenport MJ, Epps SB, Nordquist VM (2003) Developmental coordination disorder. Phys Ther 83: 722-731.

4. (2016) Centers for Disease Control and Prevention. International Classification of Diseases, Tenth Revision, Clinical Modification (ICD-10-CM).

5. Schaaf R, Miller L (2005) Occupational therapy using a sensory integrative approach for children with developmental disabilities. Ment Retard Dev Disabil Res Rev 11: 143-148.

6. Ming X, Brimacombe M, Wagner GC (2007) Prevalence of motor impairment in autism spectrum disorders. Brain Dev 29: 565-570.

7. Roley S, Bissell J, Clark G, Amini D (2015) Occupational therapy for children and youth using sensory integration theory and methods in sensory-based practice. Am J Occup Ther 69.

8. Humphries TW (1993) Clinical evaluation of the effectiveness of sensory integrative and perceptual motor therapy in improving sensory integrative function in children with learning disabilities. Occupational Therapy Journal of Research 13: 163-182.

9. Hoehn TP, Baumeister AA (1994) A critique of the application of sensory integration therapy to children with learning disabilities. Journal of Learning Disabilities 27: 338-349.

10. Han ML, Carter M, Stephenson JR (2015) Meta-analysis of Research on Sensory Integration Therapy for Individuals with Developmental and Learning Disabilities. J Dev Phys Disabil 27: 183-206.

11. Smith C, Weaver LL, Fristad MA (2015) A systematic review of sensory processing interventions for children with autism spectrum disorders. Autism 19: 133-148.

12. Watling R, Hauer S (2015) Effectiveness of Ayres Sensory Integration ${ }^{\star}$ and sensory-based interventions for people with autism spectrum disorder: a systematic review. Am J Occup Ther 69: 1-12.

13. Iwanaga R, Honda S, Nakane H, Tanaka K, Toeda H, et al. (2014) Pilot study: Efficacy of sensory integration therapy for Japanese children with high-functioning autism spectrum disorder. Occup Ther Int 21: 4-11.

14. Schaaf R, Benevides T, Mailloux Z, Faller P, Hunt J, et al. (2014) An intervention for sensory difficulties in children with autism: a randomized trial. J Autism Dev Disord 44: 1493-1506.

15. Foxe J, Molholm S, Del Bene V, Frey H, Russo N, et al. (2015) Severe multisensory speech integration deficits in high-functioning school-aged children with Autism Spectrum Disorder (ASD) and their resolution during early adolescence. Cereb Cortex 25: 298-312.

16. Gee B, Thompson K, St John H (2014) Efficacy of a Sound-based Intervention with a child with an autism spectrum disorder and auditory over-responsivity. Occup Ther Int 21: 2-20.

17. Hess E (2013) DIR /FloortimeTM: Evidence-based practice toward the treatment of autism and sensory processing disorder in children and adolescents. Int J Child Health Hum Dev 6: 267-274.

18. Wilmes B, Harrington L, Kohler-Evans P, Sumpter D (2008) Coming to our senses: Incorporating brain research findings into classroom instruction. Education 128: 659-666. 